Relations industrielles

Industrial Relations

\title{
Denis Boucher et Christian Doyon, Sachez évaluer votre personnel: le chemin de la réussite
}

\section{Denis Morin}

Volume 47, numéro 1, 1992

URI : https://id.erudit.org/iderudit/050755ar

DOI : https://doi.org/10.7202/050755ar

Aller au sommaire du numéro

Éditeur(s)

Département des relations industrielles de l'Université Laval

ISSN

0034-379X (imprimé)

1703-8138 (numérique)

Découvrir la revue

Citer ce compte rendu

Morin, D. (1992). Compte rendu de [Denis Boucher et Christian Doyon, Sachez évaluer votre personnel: le chemin de la réussite]. Relations industrielles /

Industrial Relations, 47(1), 170-173. https://doi.org/10.7202/050755ar

Tous droits réservés (C Département des relations industrielles de l'Université Laval, 1992
Ce document est protégé par la loi sur le droit d'auteur. L'utilisation des services d'Érudit (y compris la reproduction) est assujettie à sa politique d'utilisation que vous pouvez consulter en ligne.

https://apropos.erudit.org/fr/usagers/politique-dutilisation/ 
naturel) de regrouper ici les thèmes de socialisation et de culture organisationnelle.

Enfin, la sixième partie (Developing Organizational Effectiveness) semble souffrir, elle aussi, de stagnation et ne présente que peu d'éléments nouveaux ou dignes de mention. Des trois chapitres qu'elle comptait en 1973, l'édition de 1991 est réduite à deux chapitres qui traitent essentiellement du processus d'intervention en matière de développement organisationnel (Chapter 19 Organizational Development: Improving Performance) et des types d'interventions disponibles (Chapter 20 - Organizational Development Interventions). II ne reste malheureusement plus rien des préoccupations concernant le aclimat organisationnel» ou le adiagnostic organisationnel» de l'édition originale.

En somme, la valeur de cet ouvrage tient à sa transformation, malheureusement incomplète. D'un manuel hybride (management et comportement), il a été lentement mais définitivement transformé en manuel de base sur le comportement humain au travail. C'est grâce aux parties II et III qu'il prend vraiment sa valeur et que sa contribution devient appréciable. Les parties IV, $\mathrm{V}$ et VI ne semblent avoir été maintenues que pour conserver l'intégrité de la structure originale du volume et ne contribuent à l'ouvrage que parce qu'elles documentent marginalement l'effet de la structure ou des processus organisationnels sur le comportement humain.

II convient toutefois de souligner la valeur pédagogique de cet ouvrage, truffé d'exemples pratiques, de cas et d'exercices, de sommaires et de questions synthèses, de références et de notes bibliographiques. Ce manuel est conçu pour répondre aux préoccupations de tout jeune professeur (voilà pour ma nostalgie!) en quête d'une connaissance organisée, de définitions claires et d'illustrations appropriées.

Enfin, il importe de remarquer que la structure même du volume favorise une mise à jour à caractère additif. Certes, cette formule ne favorise pas l'émergence de synthèses nouvelles ou d'intégration originale de la connaissance mais ce n'est pas là, il semble, la mission de ces manuels. Comme outil pédagogique, cet ouvrage a sûrement sa place parmi tant d'autres semblables.

Alain Rondeau

École des Hautes études commerciales Montréal

Sachez évaluer votre personnel: le chemin de la réussite, par Denis Boucher et Christian Doyon, Montréal, Les Éditions Agences d'Arc, 1991, 180 p., ISBN 2-89022-254-3

L'évaluation du personnel peut se décrire comme un processus qui identifie, observe, mesure et développe la performance humaine dans les organisations. La mesure de la performance au travail a été un sujet d'intérêt et un champ d'investigation depuis l'apparition de la psychologie 
industrielle/organisationnelle. Le maintien de cet intérêt est justifié par l'importance accordée à la mesure en milieu de travail. Des critères sensibles sont nécessaires pour distinguer les divers niveaux de performance entre les individus afin de prendre des décisions dans une pluralité d'activités en gestion des ressources humaines.

Les auteurs soutiennent que l'évaluation du rendement constitue la composante la plus importante dans un système de gestion des ressources humaines. Ils accordent une signification particulière à l'évaluation du rendement puisqu'elle est intimement liée à de nombreux concepts clés en psychologie organisationnelle tels que la productivité, la motivation, le feedback et l'équité. Le livre se concentre surtout sur les caractéristiques interpersonnelles et comportementales de l'évaluation. Les aspects psychométriques de l'évaluation du rendement sont largement négligés.

Tout au long du volume, les auteurs prennent en considération la relation entre le climat organisationnel et l'évaluation du rendement. Le climat organisationnel influence l'évaluation au moyen de nombreux facteurs qui facilitent son utilisation optimale. Ils mentionnent à cet égard la communication entre le supérieur immédiat et le subordonné, la participation de l'employé ainsi que la clarification des attentes et normes associées à l'emploi. Cependant, l'instrumentalité de l'évaluation (lien performance-rémunération) de même que la légitimité (validité) du système d'évaluation du rendement sont des facteurs qui n'ont pas été adéquatement analysés. Par ailleurs, Boucher et Doyon n'offrent aucune démarche rigoureuse pour l'élaboration, l'implantation et le contrôle d'un système d'évaluation du rendement à l'intention des cadres, professionnels ou employés. A l'intérieur d'une telle démarche, la discussion se limite surtout aux méthodes d'évaluation afin de mesurer la performance au travail.

Le premier chapitre présente, sous la forme de conditions efficaces en évaluation, un modèle du processus d'évaluation du rendement. Le cadre conceptuel établit les rapports entre les variables suivantes: la motivation de l'évaluateur (conséquences de l'évaluation sur l'interaction évaluateur-évalué; l'habileté de l'évaluateur; la compréhension des procédures d'évaluation, et la détermination des normes et critères en évaluation. Les auteurs n'ont pas clairement défini l'influence de la raison d'être de l'évaluation sur ce processus. Dans la même veine, le modèle ne tente pas d'expliquer les processus cognitifs impliqués lors de l'évaluation du rendement. L'approche cognitive repose sur le postulat que le traitement humain de l'information est schématique. L'évaluateur utilise des catégories cognitives pour guider l'attention (identification), l'observation, le codage, le stockage, le rappel, l'intégration (mesure) et les actions (développement) à entreprendre dans le cadre du processus d'évaluation. Le modèle de Boucher et Doyon tient compte de l'effet de plusieurs variables contextuelles sur l'évaluation du rendement. Parmi celles-ci, les auteurs citent les politiques organisationnelles, la composition du groupe de travail et la méthode d'évaluation. La formation des évaluateurs n'est pas traitée de façon systématique. A toutes fins pratiques, le modèle du processus d'évaluation du rendement présenté dans le volume correspond dans une large mesure aux postulats théoriques du modèle de Petit et De Cotiis.

L'organisation ne doit pas seulement évaluer la performance actuelle des employés mais également analyser le potentiel de ces derniers. Les auteurs 
établissent les liens entre l'évaluation du rendement et l'évaluation du potentiel. Toutefois, les différentes techniques et approches d'évaluation associées spécifiquement à un programme d'évaluation du potentiel ne sont pas abordées si ce n'est qu'en mentionnant très rapidement les centres d'évaluation.

Le chapitre 2 se focalise sur la direction par objectifs. Elle s'avère à la fois un processus et une approche orientés vers les résultats. La direction par objectifs se doit d'être distinguée des autres méthodes d'évaluation conventionnelle. Le processus en entier est analysé. Nous pouvons relever trois grandes étapes de ce processus: (1) le supérieur et le subordonné identifient conjointement les objectifs; (2) ces objectifs définissent les principaux secteurs de responsabilités en termes de résultats attendus; et (3) les objectifs sont utilisés comme points de repère pour estimer la contribution du subordonné à l'organisation. Les auteurs démontrent qu'un tel processus réduit l'ambiguilté du rôle ainsi que l'incertitude liées aux normes de rendement. Par ailleurs, la direction par objectifs est un moyen d'accroitre l'adéquation des objectifs individuels et organisationnels. II semble ainsi primordial d'adopter aun processus en cascaden (cascading goals) pour rendre compatible les objectifs parmi les différents niveaux hiérarchiques. Les auteurs n'ont pas pleinement fait appel aux travaux de Locke et Latham sur la gestion des objectifs. Pourtant, leurs multiples recherches ont une portée pratique importante pour l'organisation.

Le chapitre 3 tente de décrire les antécédents de la performance au travail. Plus particulièrement, les auteurs conceptualisent la performance selon l'équation suivante: Rendement $=$ capacité $\times$ motivation. lis adoptent une perspective globale de la performance en empruntant plusieurs notions dans les secteurs de l'habileté humaine, la motivation, l'organisation du travail, le leadership et les processus de groupe. Nous pouvons critiquer l'équation proposée. En effet, l'équation est trop simple pour permettre une compréhension approfondie d'un phénomène aussi complexe que la performance humaine. L'équation n'inclut pas notamment la possibilité que des facteurs hors du contrôle de l'individu puissent influencer la performance. Nous pouvons ajouter à l'équation des variables d'opportunité telles que la disponibilité des ressources, les conditions de travail, les comportements du leader ainsi que les procédures au travail. Les variables d'opportunité ont un effet indirect sur la performance tandis qu'elles ont un effet direct sur la motivation et l'habileté. Boucher et Doyon ont négligé d'intégrer l'ensemble de la littérature actuelle sur les théories de la performance. Á titre d'exemple, le modèle de Wherry offre une approche psychométrique détaillée du processus d'évaluation du rendement en proposant une série d'équations mathématiques et au-delà de 46 théorèmes et corollaires. Les travaux de Blumberg et Pringle sont aussi d'un intérêt certain. Selon ces chercheurs, la performance est le produit de la capacité, de la volonté ainsi que de l'opportunité d'exécuter les tâches.

Le chapitre 4 traite de l'analyse ainsi que de la démarche pour développer les différentes méthodes d'évaluation (i.e., échelles de notation, incidents critiques, échelles basées sur les comportements). De plus, les auteurs révèlent comment elles peuvent s'intégrer dans le système d'évaluation du rendement. Les annexes constituent une extension de cette section du volume. De nombreux formulaires d'évaluation orientés vers les comportements ou les 
résultats sont présentés afin d'appliquer les notions véhiculées sur le développement des méthodes d'évaluation. Le choix de la méthode d'évaluation doit être déterminé par la raison d'être de l'évaluation. Les auteurs n'ont pas comparé les méthodes d'évaluation en fonction de: (1) leur utilisation on rapport avec les différentes activités de G.R.H.; (2) de critères quantitatís et qualitatifs d'efficacité; et (3) de leur imperméabilité à l'égard des erreurs psychométriques. Le choix de la source d'évaluation (supérieur immédiat, collègues de travail, subordonnés) ne peut être dissocié de la méthode d'évaluation. Les avantages et les inconvénients de chaque source d'évaluation sont présentés. Chacune offre une perspective particulière de la performance. Par conséquent, ces sources d'évaluation tendent à être pertinentes pour des utilisations spécifiques en évaluation. Il doit y avoir compatibilité entre la source d'évaluation et la raison d'être de l'évaluation. Les auteurs n'ont pas analysé les sources d'évaluation en fonction de la raison d'être de l'évaluation.

Le chapitre 5 décrit le processus de l'entrevue d'évaluation. Plus précisément, Boucher et Doyon discutent de la préparation, de la conduite et de la fin de l'entrevue. Par ailleurs, les auteurs traitent du style et de la motivation de l'interviewer. Ils fournissent également des recommandations quant aux comportements requis par l'interviewer. Les facteurs qui contribuent à l'efficacité de l'entrevue d'évaluation sont exposés sommairement. Les thèmes du chapitre sont analysés superficiellement. De plus, les auteurs ne discutent pas de la façon de donner du feedback négatif lors de l'entrevue et comment faire face à des situations inconfortables pendant l'évaluation.

Globalement, le livre n'est définitivement pas recommandé pour le milieu universitaire compte tenu de la faiblesse marquée du cadre théorique. Malheureusement, l'ouvrage est très incomplet pour les personnes de la pratique malgré l'intérêt que suscite le sujet.

Denis MORIN

Université Laval

Cases in Organizational Development, by Alan $M$. Glassman and Thomas G. Cummings, Homewood, IL, Richard D. Irwin, 1991, 539 p., ISBN 0256-09937-5

Issu de la dynamique de groupe et du courant des systèmes sociaux, le développement organisationnel (DO) a connu au cours des dernières années des modifications importantes au plan des cibles d'intervention sans délaisser la démarche plutôt non directive qui le caractérisait au tout début. En effet, jadis fortement centré sur l'aspect humain des organisations, le DO s'inscrit aujourd'hui dans le courant de la gestion stratégique des ressources humaines, de la culture d'entreprise en faisant de la mission, de la stratégie et des valeurs des organisations une préoccupation première au plan de lintervention.

Le présent ouvrage, qui n'est pas un traité de nature théorique, mais plutôt un recueil de cas (34 en tout), se veut un reflet des changements survenus dans ce domaine d'application des sciences du comportement et de la gestion en vue 